\title{
The roosting spatial network of a bird-predator bat
}

\author{
Miguel A. Fortuna, ${ }^{1,3}$ Ana G. Popa-Lisseanu, ${ }^{2}$ Carlos Ibáñez, ${ }^{2}$ and Jordi Bascompte ${ }^{1}$ \\ ${ }^{1}$ Integrative Ecology Group, Estación Biológica de Doñana, CSIC, Avenida M. Luisa s/n, 41013 Sevilla, Spain \\ ${ }^{2}$ Department of Evolutionary Ecology, Estación Biológica de Doñana, CSIC, Avenida M. Luisa s/n, 41013 Sevilla, Spain
}

\begin{abstract}
The use of roosting sites by animal societies is important in conservation biology, animal behavior, and epidemiology. The giant noctule bat (Nyctalus lasiopterus) constitutes fission-fusion societies whose members spread every day in multiple trees for shelter. To assess how the pattern of roosting use determines the potential for information exchange or disease spreading, we applied the framework of complex networks. We found a social and spatial segregation of the population in well-defined modules or compartments, formed by groups of bats sharing the same trees. Inside each module, we revealed an asymmetric use of trees by bats representative of a nested pattern. By applying a simple epidemiological model, we show that there is a strong correlation between network structure and the rate and shape of infection dynamics. This modular structure slows down the spread of diseases and the exchange of information through the entire network. The implication for management is complex, affecting differently the cohesion inside and among colonies and the transmission of parasites and diseases. Network analysis can hence be applied to quantifying the conservation status of individual trees used by species depending on hollows for shelter.
\end{abstract}

Key words: complex networks; epidemiology; giant noctule bat; information flow; modularity; Nyctalus lasiopterus; parasites; spatial patterns; wildlife management.

\section{INTRODUCTION}

The regular roost-switching movements of animals can be considered as channels that transport information or parasites among individuals using trees for shelter. The analysis of the roosting spatial network can trace the routes that information and diseases may follow. Some network structures allow rapid diffusion of information, whereas others can contain sections (some roosting sites) that are difficult to reach. How crucial is a tree to the transmission of information or the spreading of a disease through the roosting network? How many flows of information are disrupted or must take longer detours if a tree disappears from the network? To what extent may a tree control the flow of information between individuals due to its position in the roosting network? The spatial structure of a roosting network can provide insights into the functionality of roost changes and social grouping or segregation by describing the way that information, diseases, or parasites can travel through the network.

The presence of a dense population of roost-switching bats enclosed in a small (barely $20 \mathrm{ha}$ ) isolated roosting area provided a unique scenario in which to apply the network approach. Network analysis has been widely used in complex systems of a very different nature, such as the World Wide Web (Albert et al. 1999, 2000), the Internet (Doyle et al. 2005), the worldwide air transportation network (Guimerá et al. 2005), social net-

Manuscript received 28 January 2008; revised 30 May 2008; accepted 8 July 2008. Corresponding Editor: S. J. Schreiber.

${ }^{3}$ E-mail: fortuna@ebd.csic.es works of acquaintance (Liben-Nowell et al. 2005), scientific collaboration networks (Newman 2001), the network of human sexual contacts (Liljeros et al. 2001), metabolic networks (Jeong et al. 2000), protein networks (Jeong et al. 2001), gene regulatory networks (Luscombe et al. 2004), food webs (Paine 1966, Cohen 1978, Pimm 1982), plant-animal mutualistic networks (Bascompte et al. 2003, 2006, Jordano et al. 2003), and spatial ecological networks (e.g., Urban and Keitt 2001, Fortuna et al. 2006, Campbell Grant et al. 2007), among others. In our case, the use of trees as roosting sites by bats can also be described as a network, where trees and bats using them can be represented as two sets of nodes, and the use of a particular tree by a particular bat is indicated by a link between both the tree and bat (see Fig. 1).

Many species depend on hollows in trees for shelter and their survival can be threatened by the loss of roosting habitat (e.g., Webb and Shine 1997, Gibbons and Lindenmayer 2002). Quite often, the destruction of natural habitat forces animals to find shelter near human settlements, such as hollow trees found in public parklands. These trees are nevertheless in danger of being removed by land management agencies because of the potential danger to people from falling branches (e.g., Rhodes and Wardell-Johnson 2006, Popa-Lisseanu et al. 2008). In this case, the identification of the most important roosting locations would favor more efficient management solutions (Rhodes et al. 2006).

The largest maternity colony known worldwide of the rare giant noctule bat (Nyctalus lasiopterus), located in a heavily managed city park, is among the wildlife 


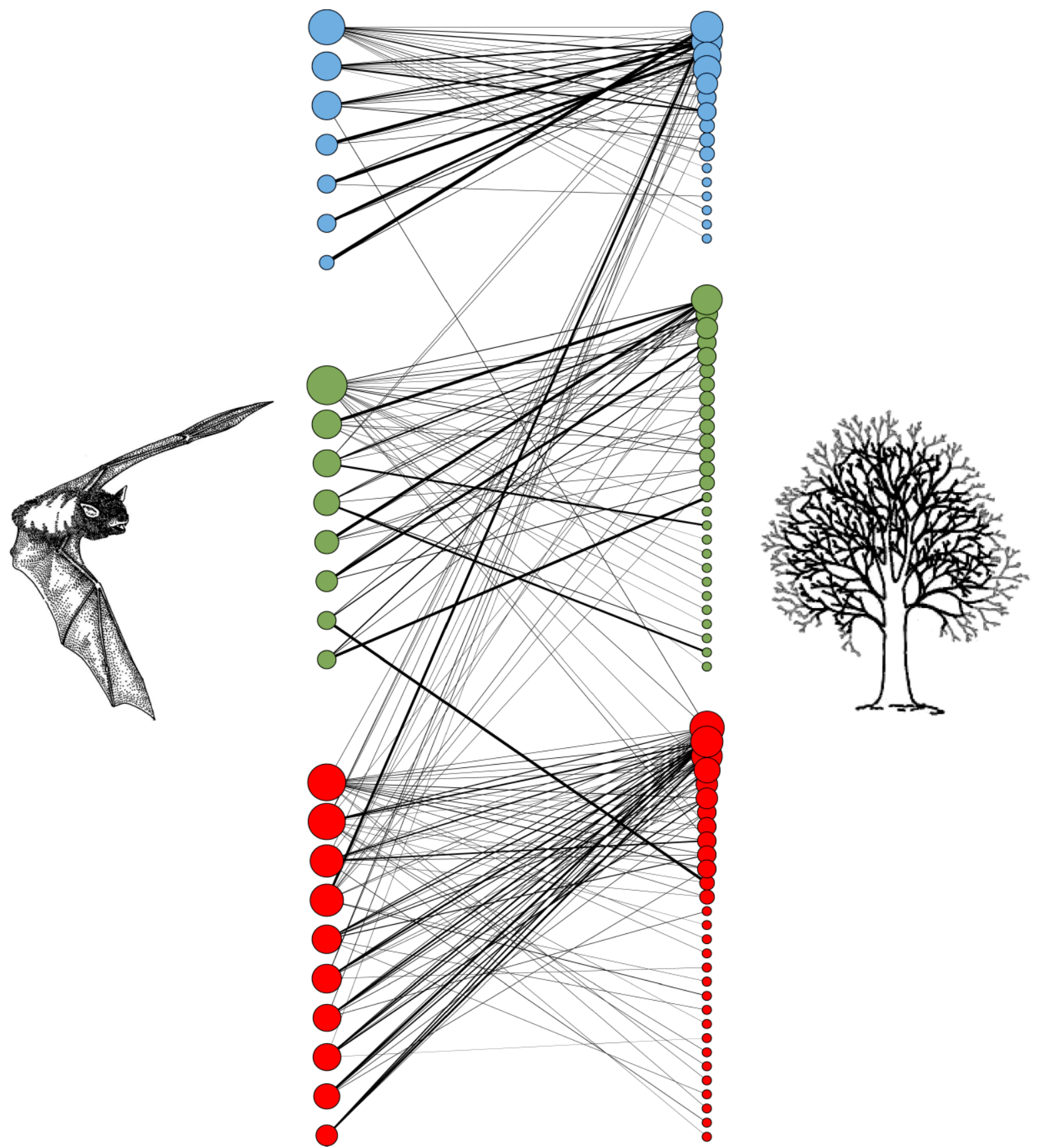

FIG. 1. Modular structure of the bipartite roosting network. Nodes represent bats $(n=25$, on the left) and trees ( $m=73$, on the right). The size of nodes is proportional (in logarithmic scale) to the number of trees visited by each bat and to the number of bats visiting each tree, respectively. A link between a bat and a tree indicates that the bat visited the tree. The thickness of a link represents the fraction of days a particular tree was visited by a particular bat from the total number of days that bat was recorded using trees. That is, it indicates how important is each tree for each bat. Colors represent the three modules detected by the algorithm, that is, three groups of bats sharing the same roosting sites and their associated three groups of trees which are used by the same bats. In blue, $n_{1}=7$ and $m_{1}=16$; in green, $n_{2}=8$ and $m_{2}=27$; in red, $n_{3}=10$ and $m_{3}=30$.

populations suffering from these threats in urban environments. A previous work on roosting dynamics of this population showed that giant noctules constitute larger stable colonies, or fission-fusion societies, spread in smaller labile roosting groups (Popa-Lisseanu et al.
2008). Bats switch tree roosts every few days so that individuals roosting in a particular tree are different from day to day. Although individuals continuously switch trees within their own colony's roosting area, colonies are stable in the long term and contact between 
nearby colonies is limited. Using now a network approach, we take up the hypotheses generated from this previous study. First, that fission-fusion societies in giant noctules can serve to maximize information transfer about roosts and perhaps foraging areas between colony members. Second, at the same time, the non-mixing of colonies within a population might preserve the own colony's information from foreigners or protect the colony from foreign diseases or parasites (Popa-Lisseanu et al. 2008).

In order to shed light on the implications that the network structure of fission-fusion dynamics has for the transmission of information, diseases, or parasites between bats spread in different trees, we have first identified modules or compartments formed by groups of bats sharing the same trees (equivalent to colonies) using a heuristic procedure from physics. Second, we have described the individual use of trees showing the heterogeneous and nested pattern in the whole network and inside each module of interlinked bats and trees. Finally, we have identified the most important trees for management from a structural (centrality measures) and dynamical (epidemiological model) approach. This importance is given by the capacity to acquire information or contract a disease, and by their role of intermediaries in the information flow or the spread of a disease through the spatial roosting network. Specifically, we will address the following questions in a broader context: does the modular and nested structure of the network explain the segregation of the population into different colonies as an effective mechanism for the isolation of these groups or the prevention of intercolony competition? Does the structure of the network enhance information transfer within colonies? Does it allow the colony to keep its knowledge safe from strangers by limiting information transfer between colonies, or can the colony avoid being infected if a neighboring colony experiences a high parasite or pathogen load?

\section{Material and Methods}

\section{Species description and study area}

The giant noctule Nyctalus lasiopterus is one of the largest and rarest Vespertilionid bats. It is unique in the animal kingdom due to its ability to predate on the wing on nocturnally migrating passerines that complement its otherwise insectivorous diet (Ibáñez et al. 2001, PopaLisseanu et al. 2007, but see Thabah et al. 2007 for another species). It appears to have a circum-Mediterranean distribution, but the species has only been found breeding in Spain and Hungary (Gombkötô et al. 1996, Ibáñez et al. 2004). The population we have studied is the largest of the species found up to date $(\sim 500$ individuals) and lives in tree cavities of a historic park in the city of Seville, southwestern Spain. Most roost trees belong to Platanus spp., Gleditsia triacanthos, Sophora japonica, and Washingtonia filifera.
Bats were monitored in 2003 and 2004. The number of days that each bat was radio tracked depended on the duration of the radio transmitter: some collars broke within a week, some lasted longer than two months. Here, we used data only for bats radio tracked at least during one month. This resulted in the roost-switching behavior of 25 adult females on 73 trees corresponding to 190 bat-tree pairs. Roosting groups at the censused trees varied between 14 to 60 individuals, but we cannot discard that some individuals could at times be roosting alone. Radio-tracked bats that have been recorded in the same tree at least one night are assumed to share the same tree in our network representation regardless of the number of times sharing simultaneously that tree. For details on the species, study area, capture and monitoring methods, patterns of roosting behavior, and social system, see Popa-Lisseanu et al. (2008).

\section{Network analysis}

We are studying a bipartite network, that is, we have established links between two sets of nodes (trees and bats) but not between nodes of the same set (see Fig. 1). We have calculated modularity (the existence of groups of bats sharing the same trees and groups of trees used by the same bats) and nestedness (a structural property where bats using a few roosting trees are a subset of the bats that use trees used by a high number of bats) on this network (Fig. 2). In order to calculate centrality measures of trees acting as roosting sites, we need to translate the so called two-mode network into a onemode network (or unipartite network) in which nodes will be now interacting trees. The procedure to build the unipartite projection of trees from the original bipartite network is as follows: any two trees that were visited by the same bat are linked (Fig. 3).

Modularity.-Modules are non-overlapping groups of nodes in networks (see Newman 2006). Community structure detection is one of the two principal lines of research for discovering them (Newman 2006). It normally assumes that the network of interest divides naturally into subgroups whose number and size are thus determined by the network itself. It also may explicitly admit the possibility that no good division of the network exists. This is the major difference and advantage with hierarchical clustering methods in which one needs to decide where to "cut" in order to obtain the relevant modules. Thus, one cannot evaluate the performance of hierarchical clustering because it does not provide a single solution to the module-identification problem (see Guimerà et al. 2007). We focused here on community structure detection as a technique allowing us to detect the existence of modules.

There are several algorithms to detect community structure in networks (see Newman and Girvan 2004, Guimerà and Amaral 2005, Rosvall and Bergstrom 2007). In this study we have used the most recent Guimerà et al.'s algorithm (Guimerà et al. 2007) for bipartite networks based on simulated annealing (Kirk- 
patrick et al. 1983). The algorithm identifies modules in each of the two sets of nodes (bats and trees) independently, as a function of the interactions shared with nodes from the other set. That is, two bats belong to the same module if they use the same roosting trees regardless of whether the trees themselves belong to the same module or not. Basically, the program uses a heuristic procedure to find an optimal solution (i.e., the maximization of a function called modularity). The modularity function for bipartite networks $M_{\mathrm{B}}$ is given by the following (see Guimerà et al. 2007):

$$
M_{\mathrm{B}}=\sum_{s=1}^{N_{\mathrm{M}}}\left[\frac{\sum_{i \neq j \varepsilon s} c_{i j}}{\sum_{a} m_{a}\left(m_{a}-1\right)}-\frac{\sum_{i \neq j \varepsilon s} t_{i} t_{j}}{\left(\sum_{a} m_{a}\right)^{2}}\right]
$$

where, for the case of bats, $N_{\mathrm{M}}$ is the number of modules, $c_{i j}$ is the number of trees used by both bats $i$ and $j, t_{i}$ and $t_{j}$ are the total number of trees used by bats $i$ and $j$, respectively, and $m_{a}$ is the number of bats using the $a$ tree. The modularity for bats (same for trees) tends to its maximum value (1) when (1) all bats visiting each tree are in the same module (first term of the equation), and (2) the probability of two randomly picked bats sharing the same tree is small (second term of the equation). Note that the sum, for all modules, of the trees shared by each pair of bats $\left[\Sigma_{s} \Sigma_{i \neq j \varepsilon s} c_{i j}\right]$ is equal to the sum, for all trees, of the number of pairs of bats sharing each tree $\left[\Sigma_{a} m_{a}\left(m_{a}-1\right)\right]$. If any tree is visited by bats belonging to different modules the sum of the first term of the equation is smaller than one. The probability of two randomly picked bats sharing the same tree is the ratio between the sum, for all modules, of the number of permutations given a fixed number of links with which each pair of bats can share a tree $\left[\Sigma_{i \neq j \varepsilon s} t_{i} t_{j}\right]$ and the maximum number of possible pairs of permutations $\left[\Sigma_{a}\right.$ $\left.\left(m_{a}\right)^{2}\right]$. Note that both denominators are global network properties which do not depend on the pair of bats considered. We tested the value of modularity for the real network with 100 random networks created keeping the same connectance (i.e., the same number of links than the real network).

Once we have identified the modules of bats and the modules of trees, we can investigate the correspondence among the modules from one set and the other (see Fig. 1). That is, we can assess the integration of each module of bats with the module of trees that they use more frequently.

Nestedness.-In order to explore the pattern of individual use of each tree by each bat inside each module, we calculate the nestedness value for the whole network and for the modules detected by the algorithm. The pattern of individual use of roosting trees by bats can be represented as a matrix, with bats as rows and trees as columns (Fig. 2). Each element of the matrix is 1 if that particular bat used that particular tree and 0 otherwise. In this presence/absence matrix, if trees are

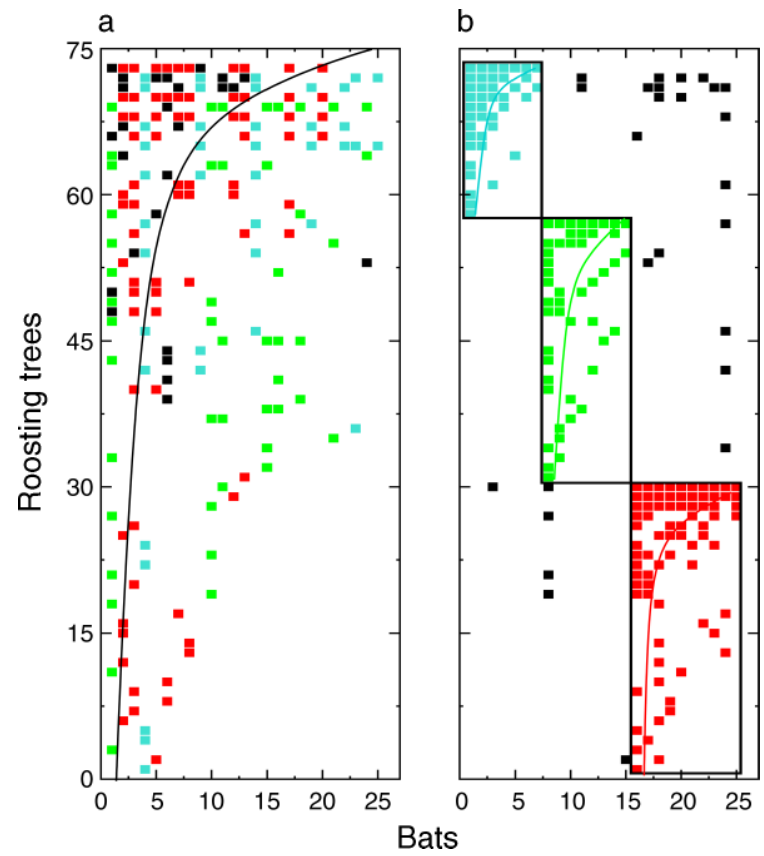

FIG. 2. (a) Binary matricial representation of the roosting network depicting trees as rows and bats as columns. A filled square indicates that a particular tree was visited by a particular bat. (b) Modular matrix version of the same network as in panel (a). Colored squares represent interactions within the same module. Each black square indicates that a particular bat and the used roosting tree belong to a different module. In the entire network (a) and inside each module (b), bats are arranged in order of decreasing number of used trees, and trees are ranked in order of increasing number of bats using them, in a way that minimizes unexpectedness. The lines represent the isoclines of perfect nestedness.

arranged in order of increasing number of bats using them, and bats are ranked in order of decreasing number of occurrences on trees, the upper left of the matrix will be filled in a roughly triangular shape.

We estimated an index of matrix nestedness $(N)$ by using the Aninhado software (Guimarães and Guimarães 2006), a modified version of the Nestedness Calculator software, originally developed by W. Atmar and B. D. Patterson (AICS Research, University Park, New Mexico, USA) to characterize how species are distributed among a set of islands (Atmar and Patterson 1993). Given the total of 73 trees, 25 bats, and 190 battree pairs defining the use of a tree by a bat, an isocline of perfect nestedness is calculated for the entire network (Fig. 2a) and for each module in the real data matrix (Fig. 2b). Absences to the left, and presences to the right of the isoclines are recorded as unexpected. For each unexpected presence or absence, a normalized measure of global distance to the isocline is calculated, and these values are averaged. By using an analogy with physical disorder, this measure is called temperature $(T)$ with values ranging from 0 to 100 (Atmar and Patterson 1993, Guimarães and Guimarães 2006). Because in this 

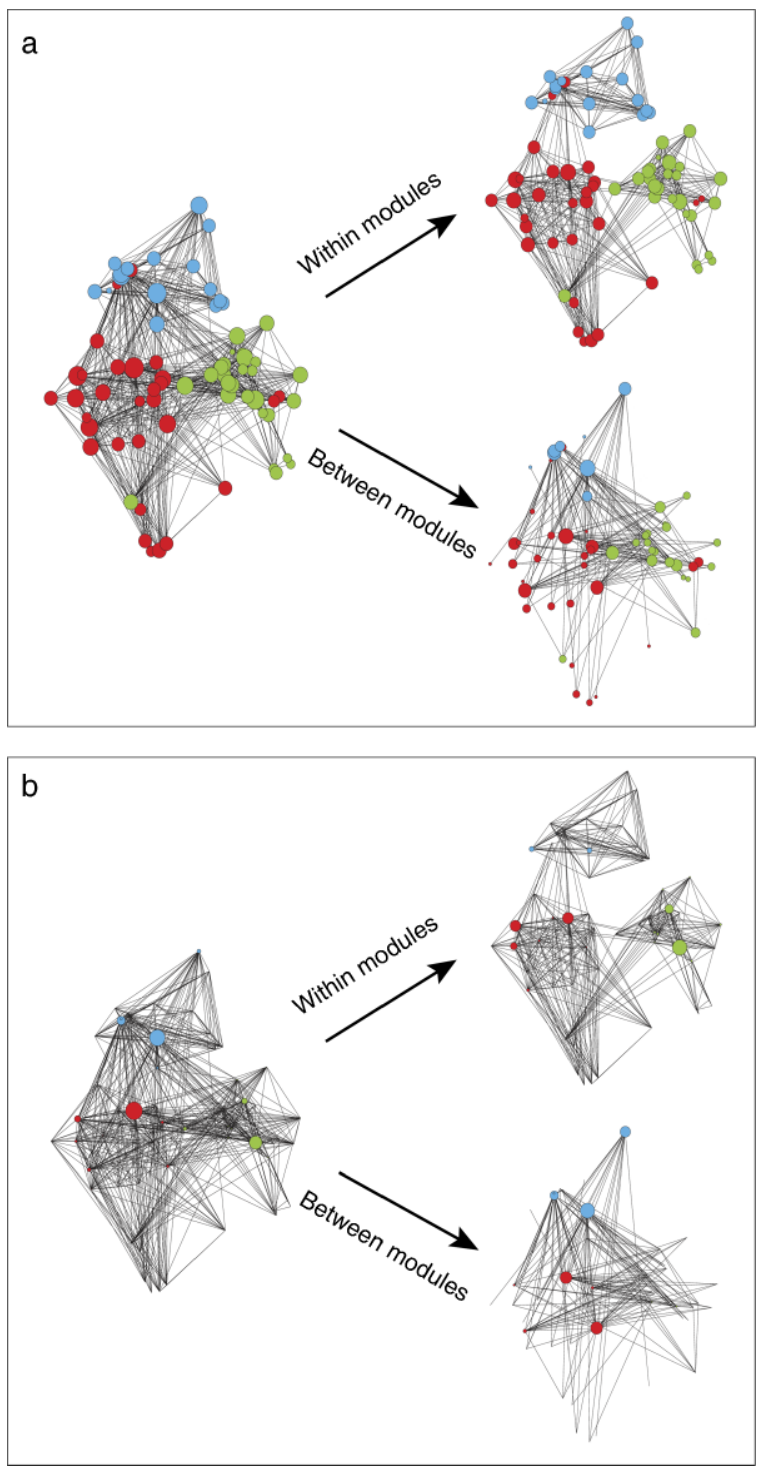

FIG. 3. Centrality analysis of the unipartite projection of roosting trees from the bipartite network of bats and trees. Nodes represent roosting trees, and their spatial distribution depicts the real spatial locations in the study area. A link between any two trees indicates that at least one bat used them once. Colors represent trees belonging to the three colonies of bats identified by the algorithm. On the left is the entire network. On the right, sub-networks showing only links between trees belonging to the same colony of bats (within modules) and between trees belonging to different colonies of bats (between modules), respectively. The size of the nodes is proportional (in logarithmic scale) to the value of the centrality measure calculated in each case.

We use a local (degree) and a global (betweenness) measure of centrality to characterize the role of a tree as a receptor and as intermediary in the flow of information or diseases through the roosting network, respectively. (a) Degree centrality, i.e., how many direct connections mediated by bats each tree has with the others. It measures, for each tree, how easy it is for a bat using that particular tree to acquire information or a disease from other bats (from the total individuals considered, on the left), from bats from the same colony (upper right), and from bats from different colonies (lower right). (b) Betweenness paper we emphasize nestedness or order instead of disorder, we define the level of nestedness, $N$, as: $N=$ $(100-T) / 100$, with values ranging from 0 to 1 (maximum nestedness; see Bascompte et al. [2003] for more details).

To assess the significance of nestedness we built the more conservative null model to contrast the observed values with the distribution of nestedness values from the 1000 resulting randomized matrices for the whole network and for each module. This null model probabilistically maintains the observed number of presences in each module. It also approximately maintains the heterogeneous distribution of the number of trees used by each bat (i.e., degree of a bat) and the number of bats which use each tree (i.e., degree of a tree) in each module. The probability of drawing a presence of bat $j$ on tree $i$ is the arithmetic mean of the presence probability of tree $i$ (i.e., the fraction of ones in column $i$ ) and bat $j$ (i.e, the fraction of ones in row $j$ ). Thus, the probability of drawing a presence is proportional to the degree of both the tree and the bat in the entire network (Fig. 2a) and in each module (Fig. 2b). For additional details on this null model, see Bascompte et al. (2003).

Centrality measures.-Using centrality measures in the unipartite projection of the trees, we focused on spatial networks as structures that allow the exchange of information or the spread of parasites and diseases. By centrality we refer to the role of individual trees in the exchange of information or the spread of parasites and diseases within the roosting network (see Fig. 3).

There are mainly three measures of the centrality of a node: degree centrality, closeness centrality, and betweenness centrality. Degree and closeness centrality are based on the reachability of a node within a network (see Fig. 3a). How easily can information reach a bat using a particular tree? A second approach to centrality rests on the idea that a node is more central if it is more important as an intermediary in the communication network. How crucial is a tree to the transmission of information or the spread of a disease through the roosting network? This approach is based on the concept of betweenness (see Fig. 3b). The betweenness centrality of a tree depends on the extent to which it is needed as a link in the chains of contacts that facilitate the spread of information or diseases within the roosting network. The more a tree is a go-between, the more central its position in the network.

In our study, we have focused on the degree centrality (Fig. 3a) and the betweenness centrality (Fig. 3b). We have calculated both measures for the entire unipartite

centrality, i.e., the proportion of all minimum distances between pairs of trees that include that particular tree. It measures how crucial a tree is for the exchange of information or the spread of a disease through the entire roosting spatial network (on the left), within the roosting network used by each colony of bats (upper right), and between the roosting network used by different colonies of bats (lower right). 
projection and also on the two subnetworks resulting, on the one hand, from considering just pairs of interlinked trees belonging to the same module, and on the other hand, from considering just pairs of interlinked trees belonging to different modules. In such a way, we have been able to separate the role of each tree as a receptor or intermediary of information or parasites/ pathogens within its own module and between modules (see Fig. 3).

The technical definition of these measures is as follows: the degree centrality of a tree is its number of links, that is, how many direct connections mediated by bats has each tree with the others. For the calculation of betweenness centrality, we previously have to define the distance between two trees as the minimum number of links needed to reach one from the other. The betweenness centrality of a tree is, hence, the proportion of all distances between pairs of other trees that include that particular tree. Some trees can be less important because if one disappears, others may fulfill its role of passing information and the communication chain between bats that used that tree remains intact.

Simulation model.- Traditional mathematical models for the spread of infectious diseases typically assume that individuals are mixed uniformly and randomly with each other (Anderson and May 1992). Recently, mathematical epidemiologists have turned to networkapproaches rejecting the homogeneous-mixing assumption and explicitly capturing the pattern of interactions among individuals (Pastor-Satorras and Vespignani 2001). In this framework, a node represents and individual host, and a link between two nodes represents an interaction that may allow disease transmission (Bansal et al. 2007).

We have developed a simulation model based on the classical susceptible-infected (SI) model from epidemiology in order to explore how rapidly information or diseases might spread via shared roosting trees. This dynamical model complements the structural properties described above. In this model there are only two classes of individuals, susceptible and infected, and once an individual becomes infected, it will not change its status thereafter. Because we are interested in the role of roosting trees we have applied the model on the bipartite projection of trees. So, in our case nodes are roosting trees and links indicate an interaction between two trees mediated by bats (i.e., both trees were visited by the same bat). The probability of a tree harboring an infected bat (hereafter, becoming an infected tree) is given by

$$
P(S \rightarrow I)=1-\left(1-p_{i}\right)^{n_{i}}
$$

where $p_{i}$ is the per contact probability at which the disease is transmitted between an infectious and susceptible tree, and $n_{i}$ is the number of infected trees linked to the susceptible tree.

By starting from one infected tree, we calculated the time steps needed for all trees becoming infected as a measure of the rate of disease spreading. We generated 1000 replicates for each starting roosting tree. We compared the mean value of these time steps with the one resulting from considering the average of 10 random networks created keeping the same number of links as in the real network. For each one of these random networks we generated 1000 replicates for each starting tree as in the real network. In both cases, we used a per contact probability $p_{i}=0.05$. Other values of this parameter do not change qualitatively the results. We have also explored the correspondence between the results obtained using structural properties and the ones resulting from the dynamical model by correlating the number of time steps with the degree and the betweeness centrality of each starting node. In order to disentangle the role of each tree within its module and between modules, we calculated the time steps to global infection within its module and across all modules. We correlated this measure of infection rate with the degree and betweeeness centrality of the starting tree within and between modules using the Spearman correlation coefficient.

\section{RESUlts}

The analysis of modularity (Fig. 1) revealed a structure significantly more modular $\left(M_{\mathrm{B}}=0.432\right.$ for bats, and $M_{\mathrm{B}}=0.390$ for trees) than expected by a random use of trees by bats $\left(M_{\mathrm{B}}=0.294 \pm 0.014\right.$ and $M_{\mathrm{B}}=0.219 \pm 0.015, P<0.001$ both for bats and trees, respectively). It also showed the existence of three welldefined groups or colonies of bats $\left(n_{1}=7, n_{2}=8\right.$, and $n_{3}$ $=10)$, and three well-defined groups of trees $\left(m_{1}=16, m_{2}\right.$ $=27$, and $m_{3}=30$ ). This result is in agreement with the three previously detected maternity colonies using hierarchical cluster analysis by Popa-Lisseanu et al. (2008). However, in a dendogram generated by hierarchical clustering, it still has to be arbitrarily decided where to "cut" in order to obtain relevant modules and hence, does not provide a single solution to the moduleidentification problem (see comments by Guimerà et al. 2007). The correspondence between modules of bats and modules of trees is clear. That is, each group of bats is associated with one group of trees maximizing the number of links inside modules $\left(q_{1}=41, q_{2}=48\right.$, and $q_{3}$ $=75$ ) and minimizing the number of links between modules $\left(q_{12}=2, q_{13}=13\right.$, and $q_{23}=11$; see also Fig. 1$)$.

The nested structure of the entire network (Fig. 2a) was higher than expected by chance $(N=0.819$ real data, $N=0.695 \pm 0.032$ null model, $P=0.001)$. The nestedness values $(N)$ of each module (Fig. 2b) were significantly higher than the ones resulting from randomizations $\left(N_{1}=0.905\right.$ real data, $N_{1}=0.673 \pm$ 0.102 null model, $P=0.004 ; N_{2}=0.766$ real data, $N_{2}=$ $0.569 \pm 0.107$ null model, $P=0.019$; and $N_{3}=0.755$ real data, $N_{3}=0.544 \pm 0.077$ null model, $\left.P=0.002\right)$. This corresponds to a scenario predicted by Lewinsohn et al. (2006). It means that bats using a few roosting trees are a subset of the bats that use trees used by a high number 


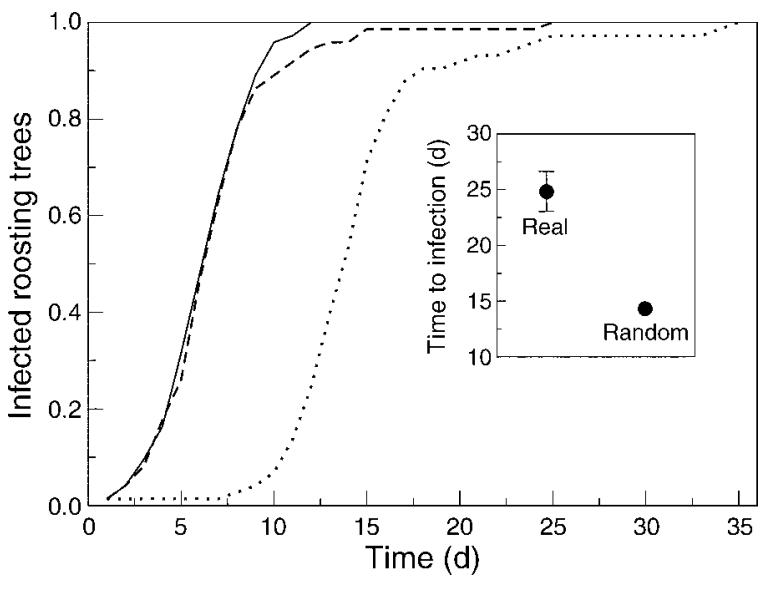

FIG. 4. Simulated spread of infection through the roosting network. Lines indicate the temporal progression of the infection (fraction of trees harboring an infected bat). Broken and dotted lines represent the spread of the infection starting from the tree with the highest and lowest degree and betweenness from the real network, respectively. The solid line indicates the spread of the infection starting from a randomly chosen tree from a random network. The inset shows the average and standard deviation of the time to global infection (all trees become infected) for the real network and for the randomization.

of bats. In the same way, trees that are used by a few bats are a subset of the trees used by bats that use many trees (Fig. 2). Besides nestedness, a heterogeneous pattern in the use of trees by bats can be observed. That is, the bulk of trees are used by a very few bats, but a few trees are much more used than expected by chance (Fig. 2). So, it seems that there is not a random pattern in the individual use of roosting trees by bats, but an heterogeneous and asymmetric hierarchical pattern through the network. Note also that only three bats (\#17, \#18, and \#25) have used trees belonging to the three modules detected (Fig. 2b).

The centrality measures of the roosting spatial network as a structure allowing the acquisition and transmission of information or diseases show contrasting results. On one hand, the degree centrality of trees indicates some heterogeneity in the role of the trees as centers of acquisition of information or diseases by the bats using them (Fig. 3a, left). If we focus on the degree centrality of trees by considering only links between trees used by the same colony of bats (within-module degree centrality), bats using any tree belonging to their colony can easily receive information from other bats belonging to the same colony (Fig. 3a, upper right). That is, all trees used by the same colony have approximately the same within-module degree centrality. But if we look at the degree centrality of trees by considering only links between trees used by bats belonging to different colonies (between-module degree centrality), only a few trees belonging to each colony of bats are useful as providers of information or parasites to bats from one colony to the others (see Fig. 3a, lower right).
On the other hand, the betweenness centrality shows a high heterogeneity in the role of trees as intermediaries in the communication network between roosting trees (Fig. 3b, left). The analysis identifies mainly three trees, one in each colony of bats, that are crucial for the transmission of information or a disease through the entire roosting spatial network. These trees are also the trees with the highest degree centrality for the entire network (see Fig. 3a, left). Note also that we can observe many trees with high degree centrality that are neither important for the control of information flow, nor facilitate the spread of a disease (several nodes are invisible because they have zero betweenness centrality: they do not mediate between other trees). But again, we can disentangle the role of trees in the information flow or the spread of a disease within and between colonies of bats by considering only links between trees used by the same colony of bats (within-module betweenness centrality, Fig. 3b, upper right) or by bats belonging to different colonies (between-module betweenness centrality, Fig. 3b, lower right), respectively. In the first case (Fig. 3b, upper right), inside one of the colonies $\left(n_{1}\right)$ there is no tree controlling the information flow. In the other two colonies, mainly two trees of each colony seem to be essential for the exchange of information or parasites to bats from one colony to the others. In the second case (Fig. 3b, lower right), one of the colonies $\left(n_{2}\right)$ does not contain any tree with this important role. On the contrary, the colony without any tree controlling the information flow inside its colony $\left(n_{1}\right)$ has two trees functioning as intermediaries in the information flow between bats belonging to other colonies. Hence, the role of a tree as a receptor (degree centrality) or as an intermediary (betweenness centrality) in the information flow or the spread of parasites or a disease depends on the spatial scale we are considering (local or global, respectively), which results from the network structure detected. Note also that most trees belonging to the same colony are near each other (Fig. 3).

The modular structure of the roosting spatial network slows down the spread of a disease or the information flow as the epidemiological simulation shows (Fig. 4). The number of time steps needed for all trees becoming infected is higher in the real network compared with a random one, even when starting the infection by the tree with the highest degree and betweenness centrality. The lower the degree and betweenness centrality of the starting tree, the longer the time to global infection. The negative correlation between the degree and betweenness centrality of trees considering the entire network and the time to global infection is significant $(\rho=-0.936, P<$ 0.001 for degree; $\rho=-0.764, P<0.001$ for betweenness). The correlation considering only links between trees belonging to the same colony is significant for degree $(\rho=-0.441, P<0.001)$ and marginally significant for betweenness $(\rho=-0.245, P=0.033)$. The correlation is also significant when considering only links between trees belonging to different colonies of 
bats $(\rho=-0.548, P<0.001$ for degree; $\rho=-0.411, P<$ 0.001 for betweenness).

\section{Discussion}

The giant noctule roosting network shows a pronounced modular structure, with three well-defined colonies of bats using three well-defined groups of trees (Figs. 1 and 2). Despite this clear modular structure, the different compartments are not totally isolated and links between them can have a very important structural function even if few in number (Lewinsohn et al. 2006). In the entire network and inside each module, specialist individuals (i.e., bats that associate with only a few trees) are a subset of the bats that associate with generalist trees (i.e., trees that host many different individuals). This asymmetric link distribution is representative of a nested pattern. This structural pattern occurs both at a population level (nestedness of the overall network) and at a colony level (nestedness within modules). The nested structure is not incompatible with the modular pattern as has been recently reported by Olesen et al. (2007) in plant-pollinator webs. The nested pattern detected inside this modular structure also implies cohesion, because bats using more trees are the ones that use the trees used by a high number of bats. This creates a core in which a small set of bats using a high number of trees uses a small set of the highly used trees. We point here a few hypotheses that could be investigated relative to the origin of this nested pattern. As all roosting trees are within very short distance of each other (distance between the two furthest trees is $550 \mathrm{~m}$ ) and giant noctules travel enormous distances from the roosts $(>40$ $\mathrm{km}$ ) for foraging (A. G. Popa-Lisseanu, F. Bontadina, and C. Ibáñez, unpublished data), all individuals should be equally able to "reach" any roost. However, some bats (generalists) could be more prone to "explore" roosts for suitability than other bats. This could reflect either differential exploratory abilities, or differential roles within the social system, where some bats could act as "scouts," exploring and gathering information in a much greater extent than others, as was suggested by Kerth and Reckardt (2003) for Bechstein's bats.

In this context, network analysis has revealed differences in the roles of individuals and their access to information in killer whale and dolphin fission-fusion societies, relative to their position in the social network (Williams and Lusseau 2006, Lusseau 2007). Alternatively, "generalist" trees could be better-quality trees (e.g., because of their physical or microclimatic characteristics, accessibility or spatial position in the network, etc.). Thus, specialist bats (which only use generalist trees) could have an advantage over generalists that spend part of their time in less-quality roosts, maybe indicating a higher rank in the society. Rank can influence the space use not only of territorial animals, but also of gregarious animals that form fission-fusion societies such as hyenas and chimpanzees (Boydston et al. 2003, Murray et al. 2007). In these cases, high- ranking individuals had smaller, more centrally located home ranges (Boydston et al. 2003) or showed more site fidelity and smaller core areas (Murray et al. 2007), comparable to a model of an ideal despotic distribution (Fretwell 1972). Likewise, "specialist" bats could be higher-ranking individuals forcing subordinates to occupy less quality trees spread across a larger area thus increasing their roost-switching rate. Unfortunately, little is known about the existence of hierarchical interactions in female bat colonies, and although suggested, such interactions have been found difficult to prove (e.g., Kerth and Reckardt 2003). Rhodes et al. (2006), illustrating roosting dynamics in forest bats, found no physical characteristics differentiating the only communal tree ("the hub") from all individually used trees, apart from its central position in the network.

The formation of fission-fusion societies in giant noctules could serve to maximize information transfer about suitable roosts or foraging areas between colony members (Popa-Lisseanu et al. 2008). On the other hand, the cost of sharing information about roosts or other valuable resources with unfamiliar or with too many individuals could explain the segregation of the population into three colonies within such a small area (Popa-Lisseanu et al. 2008). Here, we show how this maximization and control of information within and between colonies can effectively occur based on the structural properties of the network. Alternatively, differential parasite and pathogen loads between colonies could account for social separation and inter-colony avoidance in bats (Kerth et al. 2002). An important characteristic of modular structures is that they slow down the spread of anything that can travel through the network, lowering the effects of high-connectivity nodes that would otherwise rapidly act as transmitters to every portion of the network (Guimerà et al. 2007). By looking at the structural properties of the network, we can investigate how information, parasites or pathogens can be transmitted through the network, both within and between colonies. The results based on structural properties have been reinforced by the ones obtained using the dynamical simulation model. We have tracked the rate and shape of disease transmission across the roosting network, once an initial tree is "infected." The dynamical interpretation of the structural properties of the network reflects very well the underlying dynamical processes. Other studies on information transfer in animal societies based on a network approach (see the recent paper by Voelkl and Noë 2008) use dynamical models to infer how structure relates to dynamics. Therefore, our results support a strong correlation between structural and dynamical measures, further supporting the role of network structure for dynamics. The simulation model shows how the structure of the roosting network effectively slows-down the spread of information, parasites or diseases transmitted through the movements of bats between trees. It further shows that the speed of this transmission might be controlled 
through the selection of particular roosting trees, as trees with low degree and betweeness will transmit much slower than trees with high degree and betweeness, both within and between colonies. Therefore, centrality measures are useful for describing the transmission patterns within the roosting network.

Because information might lose its value with time (e.g., roosts or foraging patches becoming less suitable), we have focused on degree centrality, which takes into account only the direct connections and not those mediated by other trees (closeness centrality), as an estimate of rapid information transfer. The degree centrality of a tree represents its potential to receive information, parasites or pathogens from other trees in the network. When taking the overall network as a single unit, no clear pattern emerges. Inside each colony, the degree centrality of each tree is very alike, implying that any bat roosting anywhere in its roosting area might in the same way have access to the information that generates in any of the colony's roosts or travels through the colony. On the contrary, trees have a very heterogeneous capacity to receive information coming from a different colony. In order to receive information from members of a neighboring colony, a bat must select one of only a few specific trees (see Fig. 3a). The number of such trees differs between the three colonies, as well as their relative capacity to receive information. Colony 3 , with a few trees with very high degrees, would theoretically have a higher capacity of controlling the amount of information, parasites or pathogens received from other colonies, by positively or negatively selecting these trees. Forest bats are known to be able to detect the presence and contagiousness of parasites in roosts and to use this information when selecting day roosts. This would also help them avoid the occupation of unfamiliar roosts infected with parasites from bats that are not members of their colony (Reckardt and Kerth 2007). Bats in colony 1 , having more trees linking colonies but with a lower, more homogeneous capacity to receive information from the outside, will not have easy access to great amounts of information just by roosting in a single tree, and experience more problems to isolate themselves from another colony if the latter becomes infested with parasites.

Our second approach to centrality analysis dealt with betweeness centrality, representing the role of the trees as intermediaries in the information flow. These highly connecting nodes have the potential to act as supertransmitters to the entire network. A high heterogeneity between roosting trees is evident from a first analysis of the network (see Fig. 3b). If giant noctules, such as other forest bats, transfer information or recruit other bats at the communal roosts (Wilkinson 1992, Kerth and Reckardt 2003), high betweeness centrality trees would be the primary settings for this phenomenon. Consistent with the nested structure of the network, both generalist bats (those using many trees) and specialist bats (those using only a few) made abundant use of these roosts.
Individuals that explore little or that gather information from only a few roosting sites would thus optimize their access to information by restricting to those trees through which information must necessarily travel. While these bats would attain clear benefits from information transfer, generalists could be receiving grouping benefits (e.g., social warming) at the highbetweeness trees (see Kerth and Reckardt 2003). These mechanisms are more likely to occur within colonies, where individuals are familiar to each other (Fig. 3b). However, important differences can be observed in the three colonies: while colonies 2 and 3 have a few trees with very high-betweeness, colony 1 has no such supertransmitter trees allowing for rapid information sharing within the colony, which could be negatively affecting the cohesion of the colony. But conversely, it could have an advantage over the two other colonies if a high parasite load or a disease has entered the colony by experiencing a slower infection rate.

Very few trees control information flow between colonies, and all are different from the trees playing the same role within colonies. Through them, information, parasites and diseases can be transmitted to a neighboring colony. Popa-Lisseanu et al. (2008) suggested that the maintenance over time of three segregated colonies within such a small area could be the result of resource competition, and the need to keep the colony's knowledge, that is costly to generate and to share, only to familiar individuals that can show cooperative behaviors (see also Kerth et al. [2002] for a similar explanation of xenophobic behaviors). Each colony would then try to prevent the leakage of certain information to neighboring colonies. This could be accomplished by temporarily avoiding the inter-colony high-betweeness roosts until competitive pressures have relaxed, while the information flow within the own colony remains untouched (as within-colony highbetweeness trees are not mediators of information to other colonies).

Giant noctules have a variety of ectoparasites including mites, flees and bugs (Ibáñez et al. 2004). Although it is unlikely that parasite avoidance is the major cause of roost-switching in giant noctule colonies (Popa-Lisseanu et al. 2008; but see Reckardt and Kerth 2006, 2007), it is probably one of a variety of factors affecting it. At least one of the trees with highestbetweeness (which has also among the highest degree) was occupied continuously throughout the breeding season (Popa-Lisseanu et al. 2008). As suggested by Reckardt and Kerth (2007), some communal trees that are frequently reused, and are thus expected to have a high parasite load, could provide other advantages that outweigh the costs of heavy parasite infestation. Based on our results, we suggest that the capacity to act as primary centers for information sharing could be one of these advantages.

As we monitored only the day roosts, we cannot rule out the possibility that other processes take place during 
the night that may have important consequences for sociality (see Rhodes 2007). But our analysis clearly reveals that not all trees have the same function within the roosting network. This complicates the identification of the most-important trees for management (or trees that must be preferentially saved from logging). The implications of the removal of a tree can be complex, affecting differently the cohesion of a colony, the cohesion between colonies and the transmission of parasites and diseases, so that all factors should be taken into consideration. In general, high-degree and high-betweeness centrality trees should be preferentially protected to preserve the social interactions within and between colonies. However, in alarm situations such as the detection of a deleterious contagious disease that menaces the survival of the population, the removal of some of these highly-connected trees could be considered. The application of a network approach to study disease transmission in bat colonies using multiple roosts could therefore have important implications for conservation biology.

\section{AcKnowledgments}

During this work M. A. Fortuna and A. G. Popa-Lisseanu were funded by the Spanish Ministry of Education and Science (Fellowships BES-2004-6682 and AP-2002-3721, respectively). This work was also funded by Organismo Autónomo de Parques Nacionales (MMA), Spain, project 021/2002 (to C. Ibáñez), and by the European Heads of Research Councils, the European Science Foundation, and EC Sixth Framework Programme through a EURYI (European Young Investigator) Award (to J. Bascompte).

\section{Literature Cited}

Albert, R., H. Jeong, and A. L. Barabási. 1999. Diameter of the world-wide web. Nature 401:130-131.

Albert, R., H. Jeong, and A. L. Barabási. 2000. Error and attack tolerance of complex networks. Nature 406:378-382.

Anderson, R. M., and R. M. May. 1992. Infectious diseases of humans: dynamics and control. Oxford University Press, New York, New York, USA.

Atmar, W., and B. D. Patterson. 1993. The measure of order and disorder in the distribution of species in fragmented habitat. Oecologia 96:372-382.

Bansal, S., B. T. Grenfell, and L. A. Meyers. 2007. When individual behaviour matters: homogeneous and network models in epidemiology. Journal of the Royal Society Interface 4:879-891.

Bascompte, J., P. Jordano, C. J. Melián, and J. M. Olesen. 2003. The nested assembly of a plant-animal mutualistic network. Proceedings of the National Academy of Sciences (USA) 100:9383-9387.

Bascompte, J., P. Jordano, and J. M. Olesen. 2006. Asymmetric coevolutionary networks facilitate biodiversity maintenance. Science 312:431-433.

Boydston, E., K. Kapheim, M. Szykman, and K. Holekamp. 2003. Individual variation in space use by female spotted hyenas. Journal of Mammalogy 84:1006-1018.

Campbell Grant, E. H., W. H. Lowe, and W. F. Fagan. 2007. Living in the branches: population dynamics and ecological processes in dendritic networks. Ecology Letters 10:165-175.

Cohen, J. E. 1978. Food webs and niche space. Princeton University Press, Princeton, New Jersey, USA.

Doyle, J. C., D. L. Alderson, L. Li, S. Low, M. Roughan, S. Shalunov, R. Tanaka, and W. Willinger. 2005. The "robust yet fragile" nature of the Internet. Proceedings of the National Academy of Sciences (USA) 102:14497-14502.

Fortuna, M. A., C. Gómez-Rodríguez, and J. Bascompte. 2006. Spatial network structure and amphibian persistence in stochastic environments. Proceedings of the Royal Society B 273:1429-1434.

Fretwell, S. D. 1972. Population in seasonal environment. Princeton University Press, Princeton, New Jersey, USA.

Gibbons, P., and D. Lindenmayer. 2002. Tree hollows and wildlife conservation in Australia. CSIRO Publishing, Melbourne, Australia.

Gombkötö, P., Z. Bihari, and P. Estók. 1996. New records of the greater noctule (Nyctalus lasiopterus) and parti-coloured bat (Vespertilio murinus) in North Hungary. Denevérkutatás (Hungarian Bat Research News) 2:38-39.

Guimaraes, P. R., and P. Guimaraes. 2006. Improving the analyses of nestedness for large sets of matrices. Environmental Modelling and Software 21:1512-1513.

Guimerà, R., and L. A. N. Amaral. 2005. Functional cartography of complex metabolic networks. Nature 433: 895-900.

Guimerà, R., S. Mossa, A. Turtschi, and L. A. N. Amaral. 2005. The worldwide air transportation network: anomalous centrality, community structure, and cities' global roles. Proceedings of the National Academy of Sciences (USA) 102: 7794-7799.

Guimerà, R., M. Sales-Pardo, and L. A. N. Amaral. 2007. Module identification in bipartite and directed networks. Physical Revue E. 76:036102-1-036102-8.

Ibáñez, C., A. Guillén, and W. Bogdanowicz. 2004. Nyctalus lasiopterus (Schreber, 1780) - Riesenabendsegler. Pages 695716 in F. Krapp, editor. Handbuch der Säugetiere Europas. Volume 4. AULA-Verlag, Wiesbaden, Germany.

Ibáñez, C., J. Juste, J. L. García-Mudarra, and P. T. AgirreMendi. 2001. Bat predation on nocturnally migrating birds. Proceedings of the National Academy of Sciences (USA) 98: 9700-9702.

Jeong, H., S. P. Mason, A. L. Barabási, and Z. N. Oltvai. 2001. Lethality and centrality in protein networks. Nature 411:4142.

Jeong, H., B. Tombor, R. Albert, Z. N. Oltvai, and A. L. Barabási. 2000. The large-scale organization of metabolic network. Nature 407:651-654.

Jordano, P., J. Bascompte, and J. M. Olesen. 2003. Invariant properties in coevolutionary networks of plant-animal interactions. Ecology Letters 6:69-81.

Kerth, G., and K. Reckardt. 2003. Information transfer about roosts in female Bechtein's bats: an experimental field study. Proceedings of the Royal Society B 270:511-515.

Kerth, G., K. Safi, and B. König. 2002. Mean colony relatedness is a poor predictor of colony structure and female philopatry in the communally breeding Bechstein's bat (Myotis bechsteinii). Behavioral Ecology and Sociobiology 52:203-210.

Kirkpatrick, S., C. D. Gelatt, Jr., and M. P. Vecchi. 1983. Optimization by simulated annealing. Science 220:671-680.

Lewinsohn, T. M., P. Prado, P. Jordano, J. Bascompte, and J. M. Olesen. 2006. Structure in plant-animal interaction assemblages. Oikos 113:174-184.

Liben-Nowell, D., J. Novak, R. Kumar, P. Raghavan, and A. Tomkins. 2005. Geographic routing in social networks. Proceedings of the National Academy of Sciences (USA) 102:11623-11628.

Liljeros, F., C. R. Edling, L. A. N. Amaral, H. E. Stanley, and Y. Aberg. 2001. The web of human sexual contacts. Nature 411:907-908.

Luscombe, N. M., M. Madan Babu, H. Yu, M. Snyder, S. Teichmann, and M. Herstein. 2004. Genomic analysis of regulatory network dynamics reveals large topological changes. Nature 431:308-312. 
Lusseau, D. 2007. Evidence for social role in a dolphin social network. Evolutionary Ecology 21:357-366.

Murray, C. M., S. V. Mane, and A. E. Pusey. 2007. Dominance rank influences female space use in wild chimpanzees, Pan troglodytes: towards an ideal despotic distribution. Animal Behavior 74:1795-1804.

Newman, M. E. J. 2001. The structure of scientific collaboration networks. Proceedings of the National Academy of Sciences (USA) 98:404-409.

Newman, M. E. J. 2006. Modularity and community structure in networks. Proceedings of the National Academy of Sciences (USA) 113:8577-8582.

Newman, M. E. J., and M. Girvan. 2004. Finding and evaluating community structure in networks. Physical Review E 69:026113.

Olesen, J. M., J. Bascompte, Y. L. Dupont, and P. Jordano. 2007. The modularity of pollination webs. Proceedings of the National Academy of Sciences (USA) 104:19891-19896.

Paine, R. T. 1966. Food web complexity and species diversity. American Naturalist 100:65-75.

Pastor-Satorras, R., and A. Vespignani. 2001. Epidemic dynamics and endemic states in complex networks. Physical Revue E 63:066117.

Pimm, S. L. 1982. Food webs. Chapman and Hall, London, UK.

Popa-Lisseanu, A. G., F. Bontadina, O. Mora, and C. Ibáñez. 2008. Highly structured fission-fusion societies in an aerialhawking, carnivorous bat. Animal Behavior 75:471-482.

Popa-Lisseanu, A. G., A. Delgado-Huertas, M. Forero, A. Rodríguez, R. Arlettaz, and C. Ibáñez. 2007. Bats' conquest of a formidable foraging niche: the myriads of nocturnally migrating songbirds. PLoS ONE 2:e205.

Reckardt, K., and G. Kerth. 2006. The reproductive success of the parasitic bat fly Basilia nana (Diptera: Nycteribiidae) is affected by the low roost fidelity of its host, the Bechstein's bat (Myotis bechsteinii). Parasitology Research 98:237-243.
Reckardt, K., and G. Kerth. 2007. Roost selection and roost switching of female Bechstein's bats (Myotis bechsteinii) as a strategy of parasite avoidance. Oecologia 154:581-588.

Rhodes, M. 2007. Roost fidelity and fission-fusion dynamics of white-striped free-tailed bats (Tadarida australis). Journal of Mammalogy 88:1252-1260.

Rhodes, M., and G. Wardell-Johnson. 2006. Roost tree characteristics determine use by the white-striped freetail bat (Tadarida australis, Chiroptera: Molossidae) in suburban subtropical Brisbane, Australia. Austral Ecology 31:228-239.

Rhodes, M., G. W. Wardell-Johnson, M. P. Rhodes, and B. Raymond. 2006. Applying network analysis to the conservation of habitat trees in urban environments: a case study from Brisbane, Australia. Conservation Biology 20:861-870.

Rosvall, M., and C. T. Bergstrom. 2007. An informationtheoretic framework for resolving community structure in complex networks. Proceedings of the National Academy of Sciences (USA) 104:7327-7331.

Thabah, A., G. Li, Y. Wang, B. Liang, K. Hu, S. Zhang, and G. Jones. 2007. Diet, echolocation calls, and phylogenetic affinities of the great evening bat (Ia io; Vespertilionidae): another carnivorous bat. Journal of Mammalogy 88:728735 .

Urban, D., and T. Keitt. 2001. Landscape connectivity: a graph-theoretic perspective. Ecology 82:1205-1218.

Voelk1, B., and R. Noë. 2008. The influence of social structure on the propagation of social information in artificial primate groups: a graph-based simulation approach. Journal of Theoretical Biology 252:77-86.

Webb, J. K., and R. Shine. 1997. Out on a limb: conservation implications of tree-hollow use by a threatened snake species (Hoplocephalus bungaroides: Serpentes, Elapidae). Biological Conservation 81:21-33.

Wilkinson, G. S. 1992. Information transfer at evening bat colonies. Animal Behavior 44:501-518.

Williams, R., and D. Lusseau. 2006. A killer whale social network is vulnerable to targeted removals. Biology Letters 2: 497-500. 